\title{
Vertebrobasilar artery calcification: Prevalence and risk factors in the general population
}

\author{
Janine E. van der Toorn ${ }^{\mathrm{a}, \mathrm{b}, 1}$, Sophie R. Engelkes ${ }^{\mathrm{a}, \mathrm{b}, 1}$, M. Kamran Ikram ${ }^{\mathrm{a}, \mathrm{c}}$, M. Arfan Ikram ${ }^{\mathrm{a}}$, \\ Meike W. Vernooij ${ }^{\mathrm{a}, \mathrm{b}}$, Maryam Kavousi ${ }^{\mathrm{a}}$, Daniel Bos ${ }^{\mathrm{a}, \mathrm{b}, \mathrm{d}, *}$ \\ ${ }^{a}$ Department of Epidemiology, Erasmus MC, University Medical Centre, Rotterdam, the Netherlands \\ ${ }^{\mathrm{b}}$ Department of Radiology and Nuclear Medicine, Erasmus MC, University Medical Centre, Rotterdam, the Netherlands \\ ${ }^{c}$ Department of Neurology, Erasmus MC, University Medical Centre, Rotterdam, the Netherlands \\ ${ }^{\mathrm{d}}$ Department of Clinical Epidemiology, Harvard T.H. Chan School of Public Health, Boston, United States
}

\section{H I G H L I G H T S}

- In this large population-based study, calcification in the vertebrobasilar arteries was prevalent in $21 \%$ of the elderly.

- Calcification in the vertebrobasilar arteries was weak to moderately correlated with calcification in other arteries.

- Traditional cardiovascular risk factors were associated with vertebrobasilar artery calcification.

- Our findings suggest a different pathophysiology of arteriosclerosis in the posterior- compared to anterior circulation.

\section{A R T I C L E I N F O}

\section{Keywords:}

Vertebral artery

Basilar artery

Arterial calcification

Atherosclerosis

CT

Prevalence

Risk factors

\begin{abstract}
A B S T R A C T
Background and aims: Arteriosclerosis in the vertebrobasilar arteries may play an important role in the etiology of posterior circulation strokes, but little is known on its prevalence, its correlation with arteriosclerosis in other major arteries, and its risk factors. Hence, we investigated these aspects of vertebrobasilar artery calcification (VBAC) as marker of vertebrobasilar arteriosclerosis.

Methods: To quantify VBAC, 2483 participants (mean age: 69.2 years, 52\% female) from the Rotterdam Study underwent non-enhanced computed tomography. We determined the presence and volume of VBAC. Next, using Spearman's rank correlation, we examined the correlation between the volume of VBAC and the volume of coronary artery calcification (CAC), aortic arch calcification (AAC), and both extracranial- (ECAC), and intracranial carotid artery calcification (ICAC). Finally, we investigated associations of cardiovascular risk factors with the presence and volume of VBAC using logistic and linear regression models.

Results: The overall prevalence of VBAC was $21.0 \%$ (median volume: $7.3 \mathrm{~mm}^{3}$ [IQR: $\left.2.0-25.6\right]$ ). Correlations between VBAC and CAC, AAC, ECAC, and ICAC were weak to moderate (men: 0.33, 0.28, 0.30, 0.36; women: $0.26,0.24,0.24,0.35$, respectively). Hypertension, diabetes, and current smoking were associated with the presence of VBAC in both sexes (men: OR 1.67 [95\%-CI, 1.14-2.46], 1.60 [95\%-CI, 1.10-2.34], 1.48 [95\%-CI, 1.02-2.14]; women: OR 1.51 [95\%-CI, 1.01-2.26], 1.56 [95\%-CI, 1.02-2.39], 1.53 [95\%CI, 1.00-2.33], respectively). In men, obesity was also associated with VBAC (1.42 [95\%-CI, 1.00-2.02]).

Conclusions: VBAC occurs in over $20 \%$ of elderly community dwelling persons. Cardiovascular risk factors are associated with VBAC with similar patterns for men and women.
\end{abstract}

\section{Introduction}

Within the multifactorial etiological framework of ischemic stroke, intracranial arteriosclerosis is increasingly established as one of the most important risk factors worldwide [1,2]. Yet, important knowledge gaps pertaining to its prevalence and etiology remain. First, the vast majority of research has focused on the occurrence and risk factors of arteriosclerosis in the intracranial internal carotid artery as proxy of the

\footnotetext{
${ }^{*}$ Corresponding author. Department of Radiology and Nuclear Medicine, Department of Epidemiology, Erasmus MC, P.O. Box 2040, 3000, CA, Rotterdam, the Netherlands.

E-mail address: d.bos@erasmusmc.nl (D. Bos).

${ }^{1}$ These authors contributed equally to this work.
} 


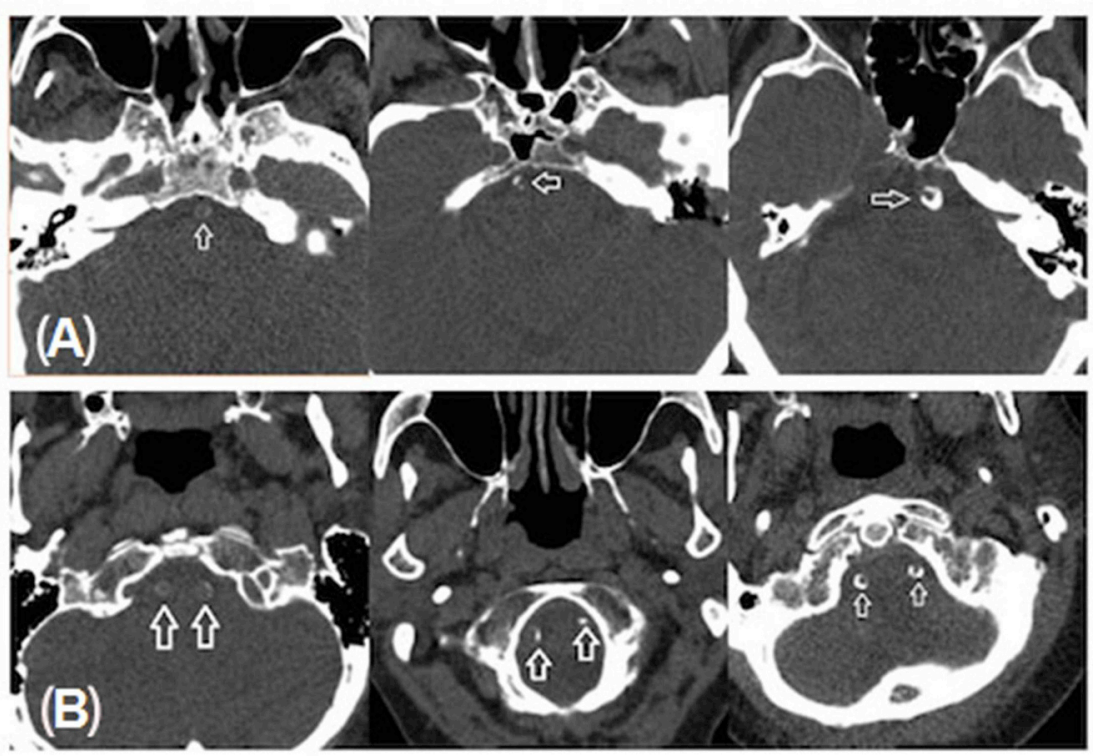

Fig. 1. Example of different calcification burden within the vertebrobasilar arteries.

anterior cerebral circulation [3,4]. Conversely, complementary data on arteriosclerosis in the posterior circulation, for which the vertebrobasilar arteries may be regarded as proxy, remain scarce [5]. However, up to a quarter of all ischemic strokes originate from the posterior circulation and may lead to a wide spectrum of symptoms ranging from vertigo or ataxia to severe locked-in syndromes [6,7]. Second, although arteriosclerosis occurs systemically across the arterial vasculature, its burden may vary considerably across arteries, with correlations ranging from 0.3 to only 0.6 [8-10]. Increasing evidence shows that this variation in arteriosclerotic burden across different arteries may be due to location-specific discrepancies in susceptibility to cardiovascular risk factors [10-13]. Additionally, differences in arteriosclerotic vulnerability to cardiovascular risk factors between men and women have been demonstrated [14]. Hence, disentangling the sex-specific associations of cardiovascular risk factors with arteriosclerosis in the vertebrobasilar arteries could aid the development of sex-specific preventive strategies for the occurrence of stroke.

Against this background, we investigated the sex-specific prevalence and distribution of vertebrobasilar calcification as a proxy for vertebrobasilar arteriosclerosis, its correlation with arteriosclerosis in other major arteries, and its risk factors in a large community-dwelling population. These novel insights will substantially advance our knowledge on the magnitude of intracranial arteriosclerosis at large and may eventually contribute to recommendations for cerebrovascular risk reduction $[15,16]$.

\section{Materials and methods}

\subsection{Study population}

This study was embedded within the Rotterdam Study, a population-based cohort ongoing since 1990 in the Ommoord district in Rotterdam, comprising over 15,000 participants. Each participant was examined at study entry and at regular follow-up examinations that were carried out every three to five years [17]. Between 2003 and 2006, participants that came to the research centre for a study round were invited to undergo a non-contrast multidetector computed tomography (MDCT) scan to visualize arterial calcification in multiple vessel beds. A total of 2524 participants (response rate: $78 \%$ ) were scanned. Due to image artifacts, vertebrobasilar artery calcification (VBAC) was not gradable in 41 participants, leaving a total of 2483 subjects for the main analyses. The Rotterdam Study has been approved by the Medical
Ethics Committee of the Erasmus MC (registration number MEC 02.1015) and by the Dutch Ministry of Health, Welfare and Sport (Population Screening Act WBO, license number 1071272-159521-PG). The Rotterdam Study has been entered into the Netherlands National Trial Register (NTR; www.trialregister.nl) and into the WHO International Clinical Trials Registry Platform (ICTRP; www.who.int/ictrp/ network/primary/en/) under shared catalogue number NTR6831. All participants provided written informed consent to participate in the study and to have their information obtained from treating physicians.

\subsection{Assessment of vertebrobasilar calcification}

We used a 16-slice $(n=781)$ or 64 -slice $(n=1702)$ multidetector CT scanner (Somatom Sensation, Siemens, Forchheim, Germany) to visualize VBAC. We assessed VBAC using a scan that ranged from the aortic root to the circle of Willis (1 $\mathrm{cm}$ above the sella turcica) $[3,13]$. We quantified calcification in the intracranial vertebral arteries from the level at which the vertebral arteries entered the dura to the level of merging into the basilar artery. Calcification in the basilar artery was assessed from the merge of the vertebral arteries to the top of the basilar artery. Detailed information on the scan protocol has been described in detail elsewhere [13].

Calcification was scored semi-automatically by drawing regions of interest (ROI) around calcification in consecutive slices in the course of the intracranial vertebral arteries and the basilar artery (Fig. 1). In the ROI, the number of pixels above 130 Hounsfield units (HU) was calculated $[3,13,18]$ and multiplied by pixel-size and the increment to determine the calcification volume in cubic millimeters, $\mathrm{mm}^{3}$. The intra- and inter-rater reliability on a random set of 50 scans were excellent (intra-rater Intraclass-Correlation Coefficient (ICC): 1.000 (95\%CI: $1.000-1.000 ; p<0.001)$ | inter-rater ICC: 0.997 (95\%-CI, 0.995-0.998; $p<0.001)$.

\subsection{Assessment of cardiovascular risk factors and calcification in other vessel beds}

Cardiovascular risk factors were assessed by means of interview, clinical examination, and blood sampling. Using the interview, we obtained information on smoking habits, and the use of lipid lowering- or blood pressure lowering medication. Smoking was categorized as 'current smoker', 'past smoker', and 'never smoker' [13,19]. We measured weight and height and calculated body mass index (BMI) [weight/ 
Table 1

Descriptive characteristics of the study population.

\begin{tabular}{|c|c|c|c|c|}
\hline & Total, $\mathrm{N}=2483$ & Men, $N=1194$ & Women, $\mathrm{N}=1289$ & $p$-value ${ }^{\mathrm{a}}$ \\
\hline Age (years) & $69.2 \pm 6.8$ & $69.3 \pm 6.7$ & $69.1 \pm 6.9$ & 0.485 \\
\hline Body mass index $\left(\mathrm{kg} / \mathrm{m}^{2}\right)$ & $27.7 \pm 4.0$ & $27.4 \pm 3.4$ & $27.9 \pm 4.5$ & 0.005 \\
\hline Obesity & $603(24.3)$ & $253(21.2)$ & $349(27.1)$ & 0.001 \\
\hline Systolic blood pressure (mmHg) & $146.6 \pm 20.3$ & $145.8 \pm 19.8$ & $147.4 \pm 20.7$ & 0.057 \\
\hline Diastolic blood pressure (mmHg) & $80.0 \pm 10.9$ & $81.0 \pm 10.9$ & $79.1 \pm 10.7$ & $<0.001$ \\
\hline Hypertension & $1825(73.5)$ & $873(73.1)$ & $952(73.9)$ & 0.787 \\
\hline Serum total cholesterol $(\mathrm{mmol} / \mathrm{l})$ & $5.7 \pm 1.0$ & $5.4 \pm 1.0$ & $5.9 \pm 1.0$ & $<0.001$ \\
\hline Serum HDL cholesterol (mmol/l) & $1.4 \pm 0.4$ & $1.3 \pm 0.3$ & $1.6 \pm 0.4$ & $<0.001$ \\
\hline Hypercholesterolemia & $1222(49.2)$ & $502(42.1)$ & $720(55.9)$ & $<0.001$ \\
\hline $\mathrm{HDL}<1.0 \mathrm{mmoL} / 1$ & $284(11.4)$ & $208(17.4)$ & $75(5.8)$ & $<0.001$ \\
\hline Serum glucose (mmol/l) & $5.7 \pm 1.3$ & $5.8 \pm 1.3$ & $5.7 \pm 1.3$ & 0.008 \\
\hline Diabetes mellitus & $332(13.4)$ & $170(14.2)$ & $161(12.5)$ & 0.115 \\
\hline Current smoking & $392(15.7)$ & $213(17.8)$ & 179 (13.9) & 0.010 \\
\hline Use of lipid lowering medication & $619(24.9)$ & $303(25.4)$ & $315(24.4)$ & 0.497 \\
\hline Use of blood pressure lowering medication & $1013(40.8)$ & $502(42.0)$ & $512(39.7)$ & 0.210 \\
\hline History of cardiovascular disease & $310(12.5)$ & $212(17.8)$ & $98(7.6)$ & $<0.001$ \\
\hline
\end{tabular}

Values are in means \pm standard deviations for continuous variables, absolute numbers and percentages for categorical variables.

${ }^{a} p$-value of characteristic differences between men and women estimated using $t$-test for continuous variables, and Chi-square test for categorical variables.

height $\left.{ }^{2}\left(\mathrm{~kg} / \mathrm{m}^{2}\right)\right]$. Accordingly, obesity was defined as a BMI of $30 \mathrm{~kg} /$ $\mathrm{m}^{2}$ or higher. Blood pressure was measured twice at the right arm in sitting position, and the average of two measurements was used. Hypertension was defined as a systolic blood pressure $\geq 140 \mathrm{mmHg}$ and/ or a diastolic blood pressure $\geq 100 \mathrm{mmHg}$ and/or the use of blood pressure lowering medication [20].

Serum total cholesterol and high-density lipoprotein (HDL) cholesterol were assessed using an automatic enzymatic procedure (Hitachi 911, Roche CHOD PAP). Hypercholesterolemia was defined as a serum total cholesterol of $\geq 6.2 \mathrm{mmoL} / \mathrm{L}$ [21] and/or the use of lipid lowering medication. We defined low HDL-cholesterol as HDL $<1.0 \mathrm{mmoL} / \mathrm{L}$ [21]. Diabetes was defined as fasting plasma glucose $\geq 7.0 \mathrm{mmoL} / \mathrm{L}$ [22] and/or the use of anti-diabetic medication. Information on history of myocardial infarction (MI), stroke, percutaneous transluminal coronary angioplasty (PCI), and coronary artery bypass graft (CABG) was collected at baseline and during follow-up visits as described earlier [23-25]. We defined history of cardiovascular disease (CVD) as history of MI, stroke, PCI, or CABG. Finally, using the CT-scans we obtained information on the volume of coronary artery calcification (CAC), aortic arch calcification (AAC), extracranial carotid artery calcification (ECAC), and intracranial carotid artery calcification (ICAC). Detailed description of the methods used for quantification of calcification in these vascular territories are provided elsewhere [13,26,27].

\subsection{Statistical analysis}

Descriptive characteristics of the study population were calculated as means with standard deviations or absolute values with percentages. Differences in population characteristics according to sex were estimated using chi-square tests for categorical variables and t-tests for continuous variables. We calculated the overall sex-specific prevalence of VBAC and examined the prevalence and median volumes of VBAC based on 10 year age strata [3,4]. Additionally, we calculated sexspecific prevalences including 95\%-confidence intervals (95\%-CI) and median calcification volumes for the left vertebral artery, right vertebral artery, and basilar artery.

Next, we examined the correlation between the volume of VBAC and the volume of CAC, AAC, ECAC, and ICAC using Spearman's rank correlation. A nonparametric bootstrap procedure with 1000 replications was performed to obtain interval estimates of Spearman's Rho. In order to provide insight into the physiological distribution of calcification in the abovementioned vessel beds, we calculated all possible combinations of affected vessels within persons [28]. For this, and for the correlation analysis, we used examinations of participants with complete information on calcification in all vascular beds $(\mathrm{N}=2,384$, men: 1130 | women: 1254).

Finally, we investigated the association of cardiovascular risk factors with the presence and volume of VBAC using the following strategy. First, using logistic regression models, we assessed the association of hypertension, diabetes mellitus, hypercholesterolemia, low HDL cholesterol, current smoking, and history of CVD with the presence of VBAC. Second, considering the skewed distribution of VBAC, we performed a natural log-transformation and added $1 \mathrm{~mm}^{3}$ to each nontransformed volume to deal with calcium scores of 0 (Ln [calcification volume $+1 \mathrm{~mm}^{3}$ ]). Subsequently, we used linear regression models to examine the association between the abovementioned risk factors and volume of VBAC. Model 1 was adjusted for age, cohort, and scanner, model 2 additionally included all cardiovascular risk factors. All analyses were stratified by sex. For missing data on cardiovascular risk factors, we used multiple imputation by chained equation [29] ( $n=5$ imputations) along with age, sex, calcification volumes and cardiovascular risk factors. None of the variables contained more than $6 \%$ of missing data. Statistical analyses were performed with IBM SPSS Statistics 24.0 (IBM, Chicago, IL, www.spss.com) and R statistical software (R-project, Institute for Statistics and Mathematics, R Core Team (2013), Vienna, Austria, version 3.4.4).

\section{Results}

\subsection{Study population}

Table 1 shows the characteristics of the study population. Our study population comprised more women than men (52\% versus $48 \%$, respectively), and the mean age ( \pm sd) was 69.2 ( \pm 6.8 ) years. BMI and cholesterol levels were higher in women than men. Men had a slightly higher diastolic blood pressure and serum glucose. The use of blood pressure and lipid lowering medication was more frequent in men than women.

\subsection{Prevalence and distribution of VBAC}

Table 2 shows the sex-specific prevalence and median volumes of vertebrobasilar calcification. The overall prevalence of VBAC was $21.0 \%$. We found a higher prevalence and larger VBAC volume in men than women, $23.7 \%$ (median volume: $10.4 \mathrm{~mm}^{3}$ [interquartile range (IQR): $2.7-28.9$ ]) vs. $18.5 \%$ (median volume: $5.4 \mathrm{~mm}^{3}$ [IQR: $1.5-17.9$ ], respectively). Calcification was more prevalent in the left vertebral artery (men: $18.3 \%$, women: $13.8 \%$ ), followed by calcification of the right vertebral artery (men: $13.8 \%$, women: $11.2 \%$ ). Calcification of the basilar artery was present in only $1.5 \%$ of the participants (men: $1.9 \%$, 
Table 2

Prevalence and volume of intracranial vertebrobasilar artery calcification.

\begin{tabular}{|c|c|c|c|c|c|c|}
\hline & \multicolumn{2}{|l|}{ Total, $\mathrm{N}=2483$} & \multicolumn{2}{|l|}{ Men, $N=1194$} & \multicolumn{2}{|l|}{ Women, $\mathrm{N}=1289$} \\
\hline & Prevalence (95\%-CI) & $\begin{array}{l}\text { Calcification volume } \\
\text { (IQR) }\end{array}$ & Prevalence $(95 \%-\mathrm{CI})$ & $\begin{array}{l}\text { Calcification volume } \\
\text { (IQR) }\end{array}$ & Prevalence $(95 \%-\mathrm{CI})$ & $\begin{array}{l}\text { Calcification volume } \\
\text { (IQR) }\end{array}$ \\
\hline Vertebrobasilar arteries & $21.0(19.4-22.6)$ & $7.3(2.0-25.6)$ & $23.7(21.3-26.1)$ & $10.4(2.7-28.9)$ & $18.5(16.3-20.6)$ & $5.4(1.5-17.9)$ \\
\hline Left vertebral artery & $16.0(14.6-17.4)$ & $5.4(1.3-18.2)$ & $18.3(16.1-20.5)$ & $8.0(1.9-24.2)$ & $13.8(11.9-15.7)$ & $2.9(0.9-12.1)$ \\
\hline Right vertebral artery & $12.4(11.2-13.7)$ & $6.4(2.2-18.5)$ & $13.8(11.9-15.8)$ & $7.0(2.3-23.4)$ & $11.2(9.5-12.9)$ & $5.9(1.7-16.9)$ \\
\hline Basilar artery & $1.5(1.1-2.0)$ & $3.1(0.6-9.7)$ & $1.9(1.2-2.7)$ & $4.0(1.4-11.7)$ & $1.2(0.6-1.8)$ & $1.0(0.5-4.7)$ \\
\hline
\end{tabular}

Values represent prevalences in percentages (95\%-confidence intervals), and median calcification volumes in mm ${ }^{3}$ (interquartile range).

Table 3

Prevalence and volume of intracranial vertebrobasilar artery calcification per age category.

\begin{tabular}{|c|c|c|c|c|}
\hline & Men, $N=1194$ & & Women, $\mathrm{N}=1289$ & \\
\hline Age categories (years) & Prevalence $(95 \%-\mathrm{CI})$ & Calcification volume (IQR) & Prevalence (95\%-CI) & Calcification volume (IQR) \\
\hline$<65$ & $13.8(10.1-17.5)$ & $12.3(2.6-30.3)$ & $10.3(7.3-13.4)$ & $3.8(0.8-10.7)$ \\
\hline $65-75$ & $23.9(20.5-27.3)$ & $9.0(2.7-28.7)$ & $16.7(13.8-19.7)$ & $4.0(1.3-17.6)$ \\
\hline $75-85$ & $34.1(27.9-40.2)$ & $9.6(2.5-27.2)$ & $31.3(25.5-37.1)$ & $8.2(2.2-26.0)$ \\
\hline$\geq 85$ & $51.9(31.7-72.0)$ & $27.2(3.5-46.4)$ & $48.6(31.2-66.0)$ & $5.4(1.9-25.3)$ \\
\hline
\end{tabular}

Values represent prevalences in percentages (95\%-confidence intervals), and median calcification volumes in mm ${ }^{3}$ (interquartile range).

women: $1.2 \%)$. In $0.8 \%$ and $0.5 \%$ of men and women, respectively, calcification was present in all three vertebrobasilar arteries (left vertebral artery, right vertebral artery and basilar artery), whereas $7.7 \%$ of the participants (men: $8.9 \%$, women: $6.7 \%$ ) had calcification in two vessels and the remaining (men: 14\%, women: $11.3 \%$ ) had single vessel calcification (Supplementary Fig. I). Table 3 shows an increasing VBAC prevalence with age, with a higher prevalence and larger volumes in men than women.

\subsection{Calcification in other vessel beds}

We found a weak correlation between the volume of VBAC and the volume CAC, AAC, ECAC, and ICAC. Spearman's correlation coefficients in men were: 0.33 (95\%-CI, 0.27-0.37), 0.28 (95\%-CI, 0.23-0.33), 0.30 (95\%-CI, 0.24-0.35), 0.36 (95\%-CI, 0.31-0.41); in women: 0.26 (95\%CI, 0.20-0.33), 0.24 (95\%-CI, 0.19-0.29), 0.24 (95\%-CI, 0.20-0.27), 0.35 (95\%-CI, 0.30-0.40).

Considering the distribution of the prevalence of calcification in different vessel beds, the most common combination was having CAC, AAC, ECAC, and ICAC in both men and women $(\mathrm{N}=520,46 \%$; $\mathrm{N}=429,34 \%$ respectively). In men, the second most common combination was having calcification in all vessels $(N=217,19 \%)$, whereas in women the second most common combination of affected vessels was the aortic arch, coronary, and intracranial carotid arteries. ( $\mathrm{N}=155,12 \%)$. Few participants had calcification in only one vessel bed (Supplementary Fig. II).

\subsection{Cardiovascular risk factors for VBAC}

Fig. 2 shows the multivariable adjusted associations between cardiovascular risk factors and the presence of VBAC according to sex. Age, hypertension, diabetes, and current smoking were associated with the presence of VBAC in both sexes (odds ratio (OR) for the presence versus absence of VBAC in men: 1.07 [95\%-CI, 1.04-1.09], 1.67 [95\%-CI, 1.14-2.46], 1.60 [95\%-CI: 1.10-2.34], 1.48 [95\%-CI: 1.02-2.14]); in women: 1.08 [95\%-CI, 1.05-1.10], 1.51 [95\%-CI, 1.01-2.26), 1.56 [95\%CI, 1.02-2.39], 1.53 [95\%-CI: 1.00-2.33]). In men, obesity was also associated with the presence of VBAC (OR: 1.42 [95\%-CI, 1.00-2.02]), whereas we found no association between obesity and VBAC in women. Similar results were found for the continuous analyses addressing differences in the natural-log-transformed VBAC volumes for each cardiovascular risk factor (Supplementary Table I).

\section{Discussion}

In this large population-based study of middle-aged and elderly participants, we found that the overall prevalence of VBAC was over $20 \%$. In men, VBAC was more prevalent and more severe than in women. We found weak correlations between the volume of VBAC and the volume of calcification in four other major vessels. Hypertension, diabetes, and current smoking were the most prominent risk factors for the presence and amount of VBAC in both sexes. Moreover, obesity was associated with VBAC among men.

In our study, the prevalence of VBAC was $21 \%$. Other studies show a varying VBAC prevalence up to $56 \%$ [30-34]. However, contrary to our population-based study, prior studies were performed among selected samples of referred patients or ischemic stroke patients, which explains the large discrepancies with our results as such populations a priori have a considerably higher chance of arteriosclerosis. The prevalence of calcification in other vascular territories has been reported within population-based settings. For example, within the Rotterdam Study, the prevalence of ICAC and CAC is approximately $82 \%$ and $85 \%$, respectively $[3,8]$. To our knowledge, our study is the first to show the prevalence and risk factors for VBAC in a non-selected population.

Considering the vertebral and basilar arteries separately, we observed a significantly higher prevalence of calcification for the left vertebral artery $(16.0 \%)$ compared with the right vertebral artery $(12.4 \%)$, whereas the basilar artery was less affected (1.5\%), which is similar to two previously reported studies investigating the distribution of VBAC among ischemic stroke patients [30,32]. On the contrary, an autopsy study from France among fatal stroke patients found that $32.7 \%$ had some degree of plaque in the basilar arteries, ranging from non-stenotic to fully occluding [31]. However, this might again be attributed to the disease staging, and to different techniques used to identify arteriosclerotic disease.

In accordance with previous studies focusing on different vascular territories $[8,10]$, we found a weak correlation between the volume of VBAC and volumes of CAC, AAC, ECAC, and ICAC in both sexes, which further underlines the existence of location-specific differences in the development of arteriosclerosis. Although the origin of these vesselspecific differences in susceptibility to arteriosclerosis requires further investigation, important explanations may be found in vessel-specific 
(A) Men

Model 1

$\begin{array}{lr}\text { Cardiovascular risk factors } & \text { OR }(\mathbf{9 5} \%-\mathbf{C l}) \\ \text { Age } & 1.07(1.05-1.09) \\ \text { Obesity } & 1.59(1.15-2.21) \\ \text { Hypertension } & 1.94(1.33-2.82) \\ \text { Diabetes } & 1.86(1.30-2.67) \\ \text { Hypercholesterolemia } & 1.33(1.01-1.76) \\ \text { HDL <1 mmol/I } & 1.15(0.80-1.65) \\ \text { Current smoking } & 1.31(0.92-1.88) \\ \text { History of CVD } & 2.33(1.68-3.22)\end{array}$

Cardiovascular risk factors
Age
Obesity
Hypertension
Diabetes

Hypercholesterolemia

$\mathrm{HDL}<1 \mathrm{mmo} / / \mathrm{l}$

Current smoking

History of CVD

\author{
OR (95\%-Cl) \\ 1.07 (1.04-1.09) \\ $1.42(1.00-2.02)$ \\ 1.67 (1.14-2.46) \\ $1.60(1.10-2.34)$ \\ $1.02(0.75-1.38)$ \\ $0.96(0.66-1.41)$ \\ $1.48(1.02-2.14)$ \\ $2.07(1.46-2.94)$
}

(B) Women

Cardiovascular risk factors
Age
Obesity
Hypertension
Diabetes
Hypercholesterolemia
HDL $<1$ mmol/l
Current smoking
History of CVD

Model 1

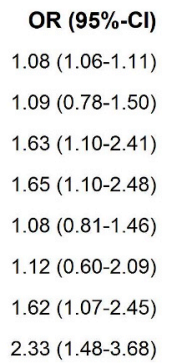

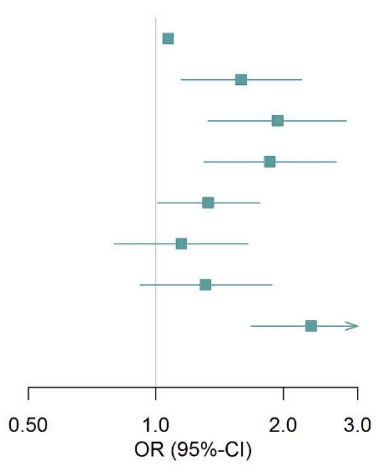

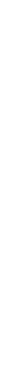

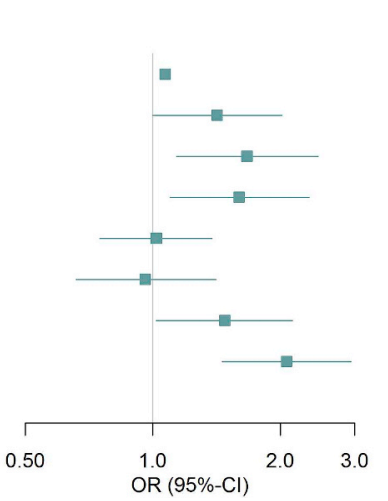

Cardiovascular risk factors
Age
Obesity
Hypertension
Diabetes
Hypercholesterolemia
HDL $<1$ mmol//
Current smoking
History of CVD
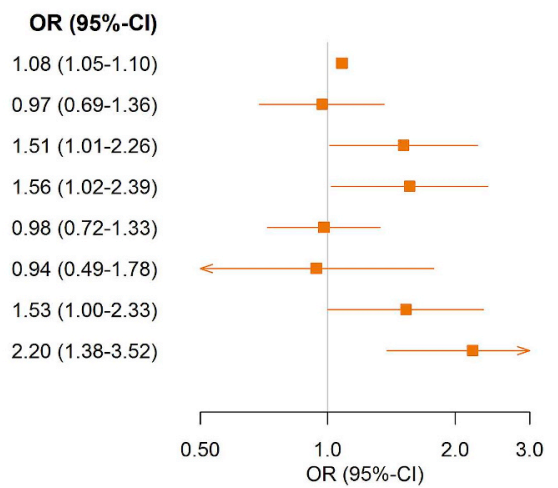

Fig. 2. Cardiovascular risk factors and the presence of vertebrobasilar calcification among (A) men and (B) women.

genetic differences $[11,35]$, or vessel-specific anatomical differences in terms of shapes, curves (turbulent flow) or diameter [36].

With regard to the association of cardiovascular risk factors with the presence and amount of VBAC, we found hypertension, diabetes, and current smoking to be most prominently related to VBAC in both sexes. Thus far, population-based research on associations between cardiovascular risk factors with calcification has been restricted to arteries other than the vertebrobasilar arteries [3,10,13,37-40]. In line with our results, diabetes, hypertension, and smoking have been identified as important risk factors for calcification in the coronary arteries, aortic arch, extracranial- and intracranial carotid arteries [3,10,13,37,39]. In addition, particularly within the Rotterdam Study, these have been identified as risk factors for arterial calcification, although the strengths of associations differed across different vessels and between women and men $[3,13]$. Within this population, it has been shown previously that smoking was an important risk factor for ICAC, AAC, and ECAC with stronger associations in men than women, whereas diabetes seems to be a strong risk factor for ECAC, AAC, and ICAC in women, but only for CAC in men. Hypertension has been identified as strong risk factor for ICAC and CAC in women, and for AAC in men $[3,13]$. In contrast with previous studies that focused on other vessel beds [3,10,13,37-40], we found no association of low HDL cholesterol with VBAC. Although this might be due to a small percentage of participants that had a low HDLcholesterol, we also found no association between hypercholesterolemia and VBAC, suggesting that the lipid-metabolism might be less prominent for the development of VBAC. Interestingly, within the same population as the current study, it has been shown that hypercholesterolemia is one of the strongest risk factors for calcification in the intracranial carotid artery [3]. Arguably, this difference could relate to distinct differences between the anatomy of the vertebrobasilar arteries and the intracranial internal carotid artery. This particular curvature of the intracranial internal carotid artery (siphon) predisposes to turbulent flow, which is known to be an important risk factor for cholesterol depositions in the arterial wall [41]. Conversely, the course of the vertebrobasilar arteries is rather smooth, without any such curves. Yet, further research into this topic is required.

Overall, our results show similar patterns of cardiovascular risk factors for VBAC among men and women. Only obesity showed to be a prominent determinant for VBAC in men, but not in women. Interestingly, prior studies reported a tendency towards an inverse association between obesity and arterial calcification in women $[3,13,40]$. A potential responsible mechanism for this might be related to estrogen production in fat mass, which could lead to, among others, favorable alterations of plasma lipoproteins and decrease in chemokines involved in monocyte migration $[42,43]$. However, some caution should be taken when interpreting this previously reported paradoxical association, considering that the underlying sex-specific pathways between obesity and arterial calcification remain poorly understood. Nevertheless, more research is necessary to better understand the sexspecific effects of cardiovascular risk factors on different locations of arteriosclerosis to improve sex-specific risk assessment strategies for stroke prevention.

Strengths of our study include the large population size and the population-based setting. However, our study also has some potential limitations. First, using CT scans to evaluate calcification in the vertebrobasilar arteries it is not possible to visualize non-calcified plaque. Nonetheless, previous evidence from autopsy studies demonstrate that calcification adequately reflects the total underlying plaque burden $[44,45]$. Yet, further studies on the interrelation of calcification, noncalcified plaque, and luminal stenosis in vertebrobasilar arteriosclerosis 
are warranted. Second, our population mainly comprised elderly persons, which may limit the generalizability of the results to a younger population. A final methodological consideration is that our relatively healthy population may contribute to a more restricted range of calcification scores, which, in turn, may potentially lead to an underestimation of the associations. Despite these limitations, in the prevention and management of stroke, one may have to consider a potential different etiology for arteriosclerosis depending on the location. In this light, it is of crucial importance to investigate the association of VBAC with the clinical development of stroke, in particular posterior circulation stroke and stroke subtypes (hemorrhagic and ischemic).

In conclusion, the findings of our study provide insight into the presence and risk factors of VBAC in a middle-aged and elderly community-dwelling population. The overall prevalence is over $20 \%$ and traditional cardiovascular risk factors are associated with VBAC, showing homogeneous patterns for men and women. Our results suggest a different pathophysiology of VBAC compared to other major vessel beds. These findings could be considered in future research concerning the etiology of posterior arteriosclerosis and might be taken into account while developing risk factor dependent approaches for cerebrovascular risk reduction.

\section{Conflicts of interest}

The authors declared they do not have anything to disclose regarding conflict of interest with respect to this manuscript.

\section{Financial support}

The Rotterdam Study is supported by Erasmus MC and Erasmus University Rotterdam; the Netherlands Organisation for Scientific Research; the Netherlands Organisation for Health Research and Development; the Research Institute for Diseases in the Elderly; the Netherlands Genomics Initiative; the Ministry of Education, Culture, and Science; the Ministry of Health, Welfare, and Sports; European Commission; and the Municipality of Rotterdam. Maryam Kavousi is supported by the VENI grant (91616079) from The Netherlands Organization for Health Research and Development (ZonMw). Daniel Bos is supported by a grant (A2017424F) from the BrightFocus Foundation. None of the funders had any role in study design; study conduct; collection, management, analysis, and interpretation of the data; and preparation, review, or approval of the article.

\section{Author contributions}

AI, KI, MV, MK, JT, SE, and DB designed and conducted the research; JT, SE, KI, AI, MV, MK and DB provided essential reagents, or essential materials; JT and SE analyzed data and performed statistical analysis; JT and SE wrote the paper; JT, SE, and DB had primary responsibility for final content; JT, SE, KI, AI, MV, MK and DB provided intellectual content to the paper; all authors have read and approved the final manuscript.

\section{Acknowledgements}

We gratefully acknowledge the contribution of the participants of the Rotterdam Study, research assistants, general practitioners, hospitals and pharmacies in Rotterdam.

\section{Appendix A. Supplementary data}

Supplementary data to this article can be found online at https:// doi.org/10.1016/j.atherosclerosis.2019.05.001.

\section{References}

[1] P.B. Gorelick, et al., Large artery intracranial occlusive disease: a large worldwide burden but a relatively neglected frontier, Stroke 39 (8) (2008) 2396-2399.

[2] A.I. Qureshi, L.R. Caplan, Intracranial atherosclerosis, Lancet 383 (9921) (2014) 984-998.

[3] D. Bos, et al., Intracranial carotid artery atherosclerosis: prevalence and risk factors in the general population, Stroke 43 (7) (2012) 1878-1884.

[4] T.T. de Weert, et al., Intracranial internal carotid artery calcifications: association with vascular risk factors and ischemic cerebrovascular disease, AJNR Am J Neuroradiol 30 (1) (2009) 177-184.

[5] L.R. Caplan, The intracranial vertebral artery: a neglected species, The Johann Jacob Wepfer Award 2012. Cerebrovasc Dis 34 (1) (2012) 20-30.

[6] G. Banerjee, S.P. Stone, D.J. Werring, Posterior circulation ischaemic stroke, Br. Med. J. 361 (2018) k1185.

[7] M. Muller-Kuppers, et al., Intracranial vertebral artery disease in the new England medical center posterior circulation Registry, Eur. Neurol. 37 (3) (1997) 146-156.

[8] A.E. Odink, et al., Association between calcification in the coronary arteries, aortic arch and carotid arteries: the Rotterdam study, Atherosclerosis 193 (2) (2007) $408-413$.

[9] D. Bos, et al., Calcification in major vessel beds relates to vascular brain disease, Arterioscler. Thromb. Vasc. Biol. 31 (10) (2011) 2331-2337.

[10] M.A. Allison, M.H. Criqui, C.M. Wright, Patterns and risk factors for systemic calcified atherosclerosis, Arterioscler. Thromb. Vasc. Biol. 24 (2) (2004) 331-336.

[11] H.H. Adams, et al., Heritability and genome-wide association analyses of intracranial carotid artery calcification: the Rotterdam study, Stroke 47 (4) (2016) 912-917.

[12] E. Lopez-Cancio, et al., Biological signatures of asymptomatic extra- and intracranial atherosclerosis: the Barcelona-AsIA (Asymptomatic Intracranial Atherosclerosis) study, Stroke 43 (10) (2012) 2712-2719.

[13] A.E. Odink, et al., Risk factors for coronary, aortic arch and carotid calcification; the Rotterdam Study, J. Hum. Hypertens. 24 (2) (2010) 86-92.

[14] C.N. Bairey Merz, et al., Insights from the NHLBI-sponsored women's ischemia syndrome evaluation (WISE) study: Part II: gender differences in presentation, diagnosis, and outcome with regard to gender-based pathophysiology of atherosclerosis and macrovascular and microvascular coronary disease, J. Am. Coll. Cardiol. 47 (3) (2006) S21-S29.

[15] Y. Li, et al., Risk factors between intracranial-extracranial atherosclerosis and anterior-posterior circulation stroke in ischaemic stroke, Neurol. Res. 39 (1) (2017) $30-35$.

[16] G. Gulli, S. Khan, H.S. Markus, Vertebrobasilar stenosis predicts high early recurrent stroke risk in posterior circulation stroke and TIA, Stroke 40 (8) (2009) 2732-2737.

[17] M.A. Ikram, et al., The Rotterdam Study: 2018 update on objectives, design and main results, Eur. J. Epidemiol. 32 (9) (2017) 807-850.

[18] A.S. Agatston, et al., Quantification of coronary artery calcium using ultrafast computed tomography, J. Am. Coll. Cardiol. 15 (4) (1990) 827-832.

[19] M.J. Bos, et al., Modifiable etiological factors and the burden of stroke from the Rotterdam study: a population-based cohort study, PLoS Med. 11 (4) (2014) e1001634.

[20] J.A. Whitworth, I.S. o.H.W.G, World Health Organization, World Health organization (WHO)/International society of hypertension (ISH) statement on management of hypertension, J Hypertens, 200321 (11) (2003) 1983-1992.

[21] Expert panel on detection, E. And A. Treatment of high blood cholesterol in, executive Summary of the third Report of the national cholesterol education program (NCEP) expert Panel on detection, evaluation, and Treatment of high blood cholesterol in adults (adult treatment panel III), Jama 285 (19) (2001) 2486-2497.

[22] A. American Diabetes, Standards of medical care in diabetes, Diabetes Care 28 (Suppl 1) (2005) S4-S36.

[23] I.M. van der Meer, et al., Predictive value of noninvasive measures of atherosclerosis for incident myocardial infarction: the Rotterdam Study, Circulation 109 (9) (2004) 1089-1094.

[24] M. Hollander, et al., Incidence, risk, and case fatality of first ever stroke in the elderly population. The Rotterdam Study, J. Neurol. Neurosurg. Psychiatry 74 (3) (2003) 317-321.

[25] M.J. Leening, et al., Methods of data collection and definitions of cardiac outcomes in the Rotterdam Study, Eur. J. Epidemiol. 27 (3) (2012) 173-185.

[26] D. Bos, et al., Intracranial carotid artery atherosclerosis and the risk of stroke in whites: the Rotterdam Study, JAMA Neurol 71 (4) (2014) 405-411.

[27] D. Bos, et al., Comparison of atherosclerotic calcification in major vessel beds on the risk of all-cause and cause-specific mortality: the Rotterdam study, Circ Cardiovasc Imaging 8 (12) (2015).

[28] A. Lex, et al., UpSet: visualization of intersecting sets, IEEE Trans. Vis. Comput. Graph. 20 (12) (2014) 1983-1992.

[29] M.J. Azur, et al., Multiple Imputation by Chained Equations: what is it and how does it work? Int. J. Methods Psychiatr. Res. 20 (1) (2011) 40-49.

[30] E. Gokcal, et al., The association of vertebrobasilar calcification with etiological subtypes, stroke recurrence and outcome in acute brainstem ischemic stroke, 52 (2) (2018) 188-193.

[31] M. Mazighi, et al., Autopsy prevalence of intracranial atherosclerosis in patients with fatal stroke, Stroke 39 (4) (2008) 1142-1147.

[32] S. Pikija, J. Magdič, T. Hojs-Fabjan, Calcifications of vertebrobasilar arteries on CT: detailed distribution and relation to risk factors in 245 ischemic stroke patients, Biomed Res Int, 2013 (2013) 918970.

[33] X.Y. Chen, et al., Intracranial artery calcification: a newly identified risk factor of 
ischemic stroke, J. Neuroimaging 17 (4) (2007) 300-303.

[34] X.Y. Chen, et al., The frequency and determinants of calcification in intracranial arteries in Chinese patients who underwent computed tomography examinations, Cerebrovasc. Dis. 21 (1-2) (2006) 91-97.

[35] D. Bos, et al., Genetic loci for coronary calcification and serum lipids relate to aortic and carotid calcification, Circ Cardiovasc Genet 6 (1) (2013) 47-53.

[36] N.K. David, BLOOD FLOW IN ARTERIES, Annu. Rev. Fluid Mech. 29 (1) (1997) 399-434.

[37] J.A. Hoff, et al., Conventional coronary artery disease risk factors and coronary artery calcium detected by electron beam tomography in 30,908 healthy individuals, Ann. Epidemiol. 13 (3) (2003) 163-169.

[38] A.B. Newman, et al., Coronary artery calcification in older adults to age 99: prevalence and risk factors, Circulation 104 (22) (2001) 2679-2684.

[39] M.A. Allison, C.M. Wright, Age and gender are the strongest clinical correlates of prevalent coronary calcification (R1), Int. J. Cardiol. 98 (2) (2005) 325-330.

[40] C. Iribarren, et al., Calcification of the aortic arch: risk factors and association with coronary heart disease, stroke, and peripheral vascular disease, Jama 283 (21) (2000) 2810-2815.

[41] C.M. Prado, et al., Turbulent blood flow plays an essential localizing role in the development of atherosclerotic lesions in experimentally induced hypercholesterolaemia in rats, Int. J. Exp. Pathol. 89 (1) (2008) 72-80.

[42] T.J. Key, et al., Body mass index, serum sex hormones, and breast cancer risk in postmenopausal women, J. Natl. Cancer Inst. 95 (16) (2003) 1218-1226.

[43] L. Nathan, G. Chaudhuri, Estrogens and atherosclerosis, Annu. Rev. Pharmacol. Toxicol. 37 (1997) 477-515.

[44] G. Sangiorgi, et al., Arterial calcification and not lumen stenosis is highly correlated with atherosclerotic plaque burden in humans: a histologic study of 723 coronary artery segments using nondecalcifying methodology, J. Am. Coll. Cardiol. 31 (1) (1998) 126-133.

[45] J.A. Rumberger, et al., Coronary artery calcium area by electron-beam computed tomography and coronary atherosclerotic plaque area. A histopathologic correlative study, Circulation 92 (8) (1995) 2157-2162. 